\title{
Why do some young cool stars show spot modulation while others do not?
}

\author{
R. J. Jackson and R. D. Jeffries \\ Astrophysics Group, Research Institute for the Environment, Physical Sciences and Applied Mathematics, Keele University, \\ Keele, Staffordshire ST5 5BG, UK
}

Accepted for publication in MNRAS

\begin{abstract}
We present far-red, intermediate resolution spectroscopy of 572 photometrically selected, low-mass stars $\left(0.2<M / M_{\odot}<0.7\right)$ in the young open cluster NGC 2516, using the FLAMES spectrograph at the Very Large Telescope. Precise radial velocities confirm membership for 210 stars that have published rotation periods from spot-modulated light curves and for another 144 stars in which periodic modulation could not be found. The two subsamples are compared and no significant differences are found between their positions in colour-magnitude diagrams, the distribution of their projected equatorial velocities or their levels of chromospheric activity. We rule out differing observational sensitivity as an explanation and conclude that otherwise similar objects, with equally high levels of chromospheric activity, do not exhibit spot-induced light curve modulation because their significant spot coverage is highly axisymmetric. We propose that the spot coverage consists of large numbers of small, dark spots with diameters of about $2^{\circ}$. This explains why about half of cluster members do not exhibit rotationally modulated light curves and why the light curve amplitudes of those that do have mean values of only 0.01-0.02 mag.
\end{abstract}

Key words: stars: rotation - stars: magnetic activity; stars: low-mass - clusters and associations: NGC 2516.

\section{INTRODUCTION}

Drawing on a solar analogy, intense dynamo-generated magnetic fields are thought to be responsible for regions of suppressed convective flux and cooler temperatures in late-type stellar photospheres (see Thomas \& Weiss 2008 and Strassmeier 2009). Evidence for dark "starspots" and their associated magnetic fields initially came from rotationally modulated broadband fluxes (Hall 1972, Eaton \& Hall 1979 and Vogt 1981), but there is now a large literature that detects and investigates starspots using direct and indirect observational techniques - Doppler imaging (CollierCameron \& Unruh 1994, Rice, Strassmeier \& Linsky 1996, Strassmeier 2002), measurements of the flux in molecular bands (e.g. Neff, O'Neal \& Saar 1995, O'Neal et al. 2004), spectroscopy of Zeeman-broadened lines (e.g. Marcy 1982, Gray 1984, Johns-Krull \& Valenti 1996) and tomography using circularly polarized light (Zeeman Doppler Imaging - Semel 1989, Donati et al. 1997).

The size, filling factor and position of spots as a function of time and as a function of stellar mass and rotation rate are important probes of the poorly understood dynamo mechanism, sometimes in physical circumstances quite unlike the Sun; for example, in stars that are very fast rotators or are fully convective. Understanding the properties of starspots is also important because they and their associated magnetic fields may be responsible for increasing the radii of low-mass stars and lowering their effective temperatures (Chabrier, Gallardo \& Baraffe 2007, Ribas et al. 2008, Jackson,
Jeffries \& Maxted 2009). Starspots complicate the interpretation of eclipsing binary and transiting planet light curves and compromise the accuracy with which fundamental stellar and planetary radii can be determined (Watson \& Dhillon 2004, Czesla et al. 2009, Morales et al. 2010). Photospheric starspots provide a nuisance source of systematic uncertainty in precision astrometric and radial velocity measurements, which may limit the detection of low-mass planets (Makarov et al. 2009, Barnes, Jeffers \& Jones 2011).

Starspots are often observed on cool members of young open clusters, usually as a means of estimating rotation periods via the rotational modulation of light curves as spots transit the visible hemisphere (van Leeuwen et al. 1987, Prosser et al. 1993). A review of the literature is provided by Irwin \& Bouvier (2009), with more recent examples including work by Irwin et al. (2009), Hartman et al. (2010), James et al. (2010), Delorme et al. (2011) and Meibom et al. (2011). Many young stars in these clusters are rapid rotators with vigorous dynamo action, leading to extensive optical, ultraviolet and X-ray manifestations of the consequent magnetic field. Spot-induced modulation is seen with a wide range of amplitudes from mmag levels up to modulations larger than 0.2 mag (peak-to-peak). Some of this diversity appears related to the expected connection between dynamo-related phenomena and rotation rate. A strong correlation has been found between modulation amplitudes and increasing rotation rate or decreasing Rossby number (the dimensionless ratio of rotation period to convective 
turnover time; O'Dell et al. 1995, Messina 2001). Nevertheless, even at a given rotation rate or Rossby number there is still a wide range of light curve variability. Sometimes rotational modulation is seen in a large fraction of studied cluster members. For example, Irwin et al. (2008) found rotation periods for about 50 per cent of members in the young ( $35 \mathrm{Myr}$ ) cluster NGC 2547, and Hartman et al. (2010) found rotation periods for 74 per cent of lowmass Pleiades (age $120 \mathrm{Myr}$ ) members. However, in other studies this fraction can be very much lower. A study of low-mass stars in Praesepe (age $600 \mathrm{Myr}$ ) found rotation periods for only $\sim 10$ per cent of members (Agüeros et al. 2011).

Stars where rotation periods cannot be measured may offer important clues to the dynamo mechanism or the surface distribution of starspots, and their dependence on mass and rotation rate. Alternatively, the non-detections may simply be a result of observational biases in sensitivity and sampling. When using measured rotation periods to investigate angular momentum evolution it is important to understand whether the observed samples with detected rotation rates are biased according to their rotation rates. For example, slower rotators may have smaller spot-induced light curve variations that evade detection.

In this paper we take advantage of a homogeneous survey for rotation periods undertaken in the young $(150 \mathrm{Myr})$ open cluster NGC 2516 by Irwin et al. (2007, hereafter IHA07). In previous work (Jackson et al. 2009, Jackson and Jeffries 2010) we presented spectroscopic observations of the low-mass $\left(0.2<M / M_{\odot}<0.7\right)$ stars in NGC 2516 with detected rotational modulation and period estimates from IHA07. We measured projected equatorial velocities and chromospheric activity and showed that these rapidly rotating stars were highly magnetically active and likely to have large filling factors of dark starspots. Here we extend this study to a large sample of photometric candidates in NGC 2516 that did not have measured periods in an effort to understand why some low-mass stars exhibit rotational light curve modulation and some do not.

Section 2 describes the spectroscopy and determination of radial velocity (RV), projected equatorial velocity $(v \sin i)$ and chromospheric activity. In section 3 the RV data are used to determine a list of cluster members and measure the proportion of stars with and without measured rotational periods. In section 4 we compare the measured properties of these two groups of stars. Finally, in section 5 we discuss what form of spot distribution could give rise to the observed properties of fast rotating, low-mass stars in NGC 2516 and how this distribution might affect other methods of observing starspots on young stars.

\section{SPECTROSCOPIC OBSERVATIONS OF M-DWARFS IN OPEN CLUSTER NGC 2516}

NGC 2516 is a relatively close and well studied, young open cluster. Recent papers describe membership surveys and characterisation of the cluster mass function (Jeffries, Thurston \& Hambly 2001; Sung et al. 2002; Moraux, Bouvier \& Clark 2005). The age of the cluster has been determined as $\simeq 150 \mathrm{Myr}$ from the nuclear turn off in high mass stars and the lithium depletion and X-ray activity seen in cooler stars (Jeffries, James \& Thurston 1998; Lyra et al. 2006). Metallicity is close to solar; determined spectroscopically as $[\mathrm{Fe} / \mathrm{H}]=0.01 \pm 0.07$ and photometrically as $[\mathrm{M} / \mathrm{H}]=-0.05 \pm 0.14$ (Terndrup et al. 2002). The same authors give an intrinsic distance modulus of $7.93 \pm 0.14(385 \pm 25 \mathrm{pc})$ based on main sequence fitting and a cluster reddening of $E(B-V)=0.12 \pm 0.02$. These values of distance modulus and reddening are used in what follows.
Table 1. Details of observing programme 380.D-0479

\begin{tabular}{cllccc}
\hline $\begin{array}{c}\text { Ref No. } \\
\text { Run No. }\end{array}$ & $\begin{array}{l}\text { Date \& } \\
\text { start time }\end{array}$ & $\begin{array}{l}\text { Centre } \\
\text { RA / Dec }\end{array}$ & $\begin{array}{c}\text { Seeing } \\
\text { start/end }\end{array}$ & $\begin{array}{c}\text { No. of } \\
\text { targets }\end{array}$ & $\begin{array}{c}\text { with } \\
\text { period }\end{array}$ \\
\hline 287508 & $27-11-07$ & 118.639 & 0.78 & $108(108)$ & $45(45)$ \\
1 & $04: 57$ & -60.804 & 0.79 & & \\
287510 & $27-11-07$ & 118.209 & 0.91 & $103(101)$ & $22(21)$ \\
2 & $05: 53$ & -61.107 & 0.75 & & \\
287512 & $27-11-07$ & 119.102 & 0.69 & $112(110)$ & $44(43)$ \\
3 & $06: 48$ & -61.108 & 0.79 & & \\
repeat & $27-11-07$ & 119.102 & 0.91 & $112(0)$ & $44(0)$ \\
4 & $07: 39$ & -61.108 & 0.89 & & \\
287514 & $02-01-08$ & 120.010 & 1.15 & $110(110)$ & $38(38)$ \\
5 & $06: 25$ & -61.265 & 1.03 & & \\
287516 & $29-11-07$ & 119.804 & 1.19 & $111(104)$ & $55(50)$ \\
6 & $06: 51$ & -60.934 & 1.30 & & \\
287518 & $02-01-08$ & 120.594 & 1.15 & $102(102)$ & $41(41)$ \\
7 & 05.31 & -60.790 & 1.16 & & \\
287520 & $30-12-07$ & 120.097 & 0.94 & $112(110)$ & $39(38)$ \\
8 & $02: 40$ & -60.549 & 0.85 & & \\
287522 & $30-12-07$ & 119.462 & 1.21 & $111(83)$ & $32(22)$ \\
9 & $01: 44$ & -61.402 & 1.21 & & \\
\hline
\end{tabular}

The field centres are in degrees and the seeing in arcsec.

Values in brackets indicate the number of new targets observed per OB.

Previous observations of chromospheric and coronal activity of late $\mathrm{K}$ and M-dwarfs in NGC 2516 are summarised in Jackson and Jeffries (2010, hereafter JJ10).

IHA07, surveyed NGC 2516 as part of a larger monitoring survey of young open clusters (Hodgkin et al. 2006; Aigrain et al. 2007). They identified about 1000 potential cluster members from a $V$ versus $V-I$ colour magnitude diagram (CMD), selecting targets with apparent magnitudes in the range $16<V<26$ that are close to an empirically defined isochrone. Periods were reported for 362 candidate members in the mass range $0.15-0.7 M_{\odot}$.

\subsection{Target selection and observations}

Targets for our spectroscopic survey were selected from IHA07 with $14<I<19$. They were observed using the European Southern Observatory (ESO) $8.2 \mathrm{~m}$ aperture Very Large Telescope (UT-2 Kueyen) FLAMES fibre instrument, feeding the Giraffe and UVES spectrographs. Multiple targets were observed with Giraffe in eight separate fibre configurations. The allocation of fibres to targets was biased in the sense that targets with measured periods in IHA07 were placed first and the remaining fibers allocated to candidate members from IHA07 without measured periods. About 15 fibres in each configuration were reserved for "blank" sky positions.

The Giraffe spectrograph was used with the HR20A grating, covering wavelengths $8060-8600 \AA$ at a resolving power of 16000 . Bright, early type stars in NGC 2516 were selected from the catalogue of Dachs and Kabus (1989) for use as telluric standards. Several of these stars in each configuration were observed with UVES at a resolving power of 47000 . Details of the eight configurations are shown in Table 1 and their spatial locations indicated in Fig. 1. Each configuration was observed with two sequential 1280 s Giraffe exposures and three sequential 800 s UVES exposures. One configuration was observed twice during the same night. The repeated data were useful in assessing measurement uncertainties. A total of 981 spectra were recorded for 824 unique targets. 


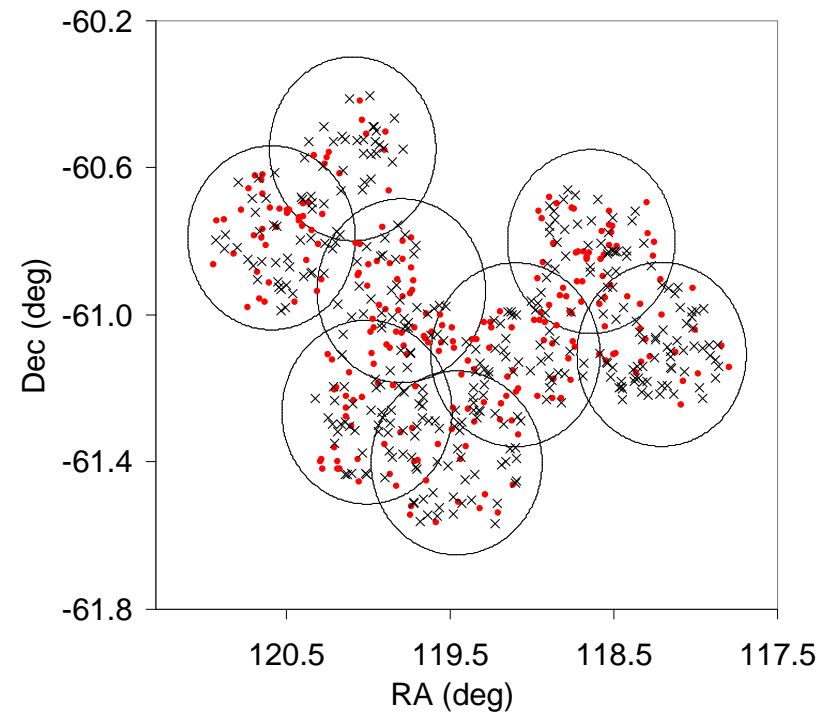

Figure 1. The distribution of targets in NGC 2516 (see Table 1). Circles define the boundaries of the eight FLAMES fields. Filled circles show target stars with a spectral signal-to-noise ratio (SNR) $\geqslant 5$, and measured period. Crosses show targets with a SNR $\geqslant 5$ but no period.

\subsection{Radial and projected equatorial velocities}

Spectra were optimally extracted and analysed using the pipeline described in detail by $\mathrm{JJ} 10$. RVs and $v \sin i$ were determined by convolving the spectra of targets with those of standard stars over the wavelength range $8061 \AA$ to $8530 \AA$, excluding the chromospherically contaminated Ca II infra-red triplet (Ca II IRT) components at $8498 \AA$ and $8542 \AA$. Templates of type K4.5V (HD 209100) and M6V (HD 34055) from the UVES atlas (Bagnulo et al. 2003) were used, bracketing the range of target spectral types. For spectra with a signal-to-noise ratio per extracted pixel $(\mathrm{SNR}) \geqslant 5$, there was usually a clearly defined single peak in the cross correlation function (CCF) giving an unambiguous value of RV and $v \sin i$. For spectra with $\mathrm{SNR}<5$, the CCF peak was often distorted by random noise and these results were considered unreliable and rejected from the sample. Spectra with SNR $\geqslant 5$ were obtained for 588 stars. Sixteen of these still showed distorted CCF peaks that did not yield an RV or $v \sin i$ (see Table 2).

Uncertainties in RV and $v \sin i$ were determined empirically by comparing repeated measurements for a subset of targets (see JJ10 for details). This gave relationships for uncertainties in RV and CCF width, $W$, as a function of SNR and $v \sin i$;

$$
\begin{aligned}
& \sigma_{\mathrm{RV}}=\sqrt{\left(9.2+0.013(v \sin i)^{2}\right)^{2} / \mathrm{SNR}^{2}+0.31^{2}} \mathrm{~km} \mathrm{~s}^{-1} \\
& \sigma_{W}=\sqrt{\left(8.8+0.005(v \sin i)^{2}\right)^{2} / \mathrm{SNR}^{2}+0.40^{2}} \mathrm{~km} \mathrm{~s}^{-1}
\end{aligned}
$$

The uncertainty in $v \sin i$ was determined from $W$ and the "zero velocity width", $K$ for each standard (where $K=19.1 \pm$ $0.6 \mathrm{~km} \mathrm{~s}^{-1}$ for the K4.5 standard and $K=17.7 \pm 0.5 \mathrm{~km} \mathrm{~s}^{-1}$ for the M6 standard). Taking $v \sin i \propto(W-K)^{1 / 2}$ we obtain

$\sigma_{v \sin i}=v \sin i \sqrt{\sigma_{W}^{2}+\sigma_{K}^{2}} /[2(W-K)] \mathrm{kms}^{-1}$.

\subsection{Chromospheric activity indices}

The spectra include two of the Ca II IRT lines with rest wavelengths of $8498 \AA$ and $8542 \AA$. These lines are known to be effective chro-
Table 2. Categorisation of targets with SNR $\geqslant 5$ and unresolved RV. The coordinates and $I$ magnitudes are from IHA07, with $I$ corrected as described

\begin{tabular}{|c|c|c|c|c|}
\hline $\begin{array}{l}\text { RA } \\
(\mathrm{J} 2000)\end{array}$ & $\begin{array}{l}\text { Dec } \\
(\text { J2000) }\end{array}$ & $\begin{array}{l}\text { Apparent } \\
I \text { mag. }\end{array}$ & SNR & $\begin{array}{l}\text { Category } \\
\text { (see notes) }\end{array}$ \\
\hline 75654.04 & -612959.48 & 15.08 & 28 & Broad peak \\
\hline 75839.38 & -613340.48 & 17.48 & 5 & Possible binary \\
\hline 75622.44 & -612129.30 & 17.87 & 5 & Low SNR \\
\hline 75628.62 & -611711.80 & 15.00 & 37 & Broad peak (p) \\
\hline 75717.64 & -611531.00 & 17.85 & 6 & Low SNR \\
\hline 75846.35 & -611431.95 & 17.01 & 6 & Low SNR \\
\hline 75619.26 & -610737.25 & 18.35 & 5 & Low SNR \\
\hline 75656.36 & -610057.55 & 14.77 & 44 & Broad peak \\
\hline 80058.89 & -612504.30 & 16.95 & 12 & Probable binary \\
\hline 75955.73 & -610808.80 & 15.76 & 29 & Broad peak \\
\hline 75932.60 & -605907.50 & 15.36 & 26 & Broad peak (p) \\
\hline 80311.28 & -603422.24 & 15.57 & 22 & Broad peak \\
\hline 75205.85 & -605655.33 & 18.28 & 5 & Possible binary \\
\hline 75332.20 & -610755.22 & 15.17 & 36 & Broad peak \\
\hline 7546.64 & -604109.90 & 15.71 & 27 & Probable binary $(\mathrm{p})$ \\
\hline 75423.11 & -604434.46 & 15.65 & 18 & Probable binary \\
\hline
\end{tabular}
in section 2.4 .

Probable binary- clear double peak in the CCF for high SNR.

Possible binary - apparent double peak in the CCF for low SNR.

Low SNR - distorted peak in the CCF for low SNR.

Broad peak - CCF indicative of a fast rotator.

(p) period reported in IHA07

mospheric activity indicators, with chromospheric emission filling the underlying absorption lines (Mallik 1994, 1997). The spectral subtraction technique used to measure the equivalent width of the Ca II IRT lines, follows that of Marsden, Carter and Donati (2009) and is detailed in JJ10. The conversion between equivalent width (EW) and activity index, $R_{C a}^{\prime}$, (the ratio of chromospheric Ca II IRT flux, corrected for any photospheric contribution, to the bolometric flux), was established using continuum flux densities and colours tabulated by Pickles (1998).

\subsection{Tabulated results}

Results for the 572 targets with SNR $\geqslant 5$ and a resolved RV are given in Tables 3 and 4. In Table 3 the identifier, RA and Dec and period (when measured) are taken from IHA07. The $V$ and I magnitudes were based on those given by IHA07, but corrected to put them onto the better calibrated photometric scale of Jeffries et al. (2001) using stars common to both papers. The corrections (added to the IHA07 values) were $\Delta I=0.080-0.0076 I$ and $\Delta(V-I)=0.300-0.153(V-I) . K$ magnitudes from the Twomicron All-Sky Survey (2MASS) survey (Cutri et al. 2003) were transformed to the CIT system using $K_{\mathrm{CIT}}=K_{2 \mathrm{MASS}}+0.024$ (Carpenter 2001). No match to the 2MASS catalogue was found for 4 targets. RVs are shown relative to the average cluster RV, estimated using the RVs of targets with $v \sin i<20 \mathrm{~km} \mathrm{~s}^{-1}$ (see section 3.1). A minimum resolvable value of $8 \mathrm{~km} \mathrm{~s}^{-1}$ is taken for $v \sin i$. At this level of broadening the increase in CCF width is $\simeq 1.3$ times the standard deviation of the "zero rotation" width; hence there is $\mathrm{a} \simeq 90$ percent probability that $v \sin i$ is truly non-zero. For targets with repeated measurements Table 3 shows weighted means of RV and $v \sin i$ with appropriate errors.

Table 4 gives Ca II IRT EWs and activity indices. Also shown are the first harmonic amplitudes for stars with rotational periods (from IHA07). The luminosity of the targets $\left(\log _{10} L / L \odot=\right.$ 
Table 3. Velocity data measured for targets in NGC 2516. The identifier, co-ordinates and rotation period are taken from Table 1 of IHA07. $V$ and $I$ photometry are also from IHA07, but corrected in the way described in section 2.4. $K$-band photometry comes from 2MASS, transformed to the CIT system. Relative RV and $v \sin i$ values are given for 572 targets which have a spectral $\mathrm{SNR} \geqslant 5$, of which 354 are identified as cluster members. The right hand column indicates the run number(s) for the observation (see Table 1), an asterisk indicates a non-member. The full table is available on Blackwell Synergy as Supplementary Material to the on-line version of this paper.

\begin{tabular}{lllllllllll}
\hline Identifier & $\begin{array}{l}\text { RA } \\
(\mathrm{J} 2000)\end{array}$ & $\begin{array}{l}\text { Dec } \\
(\mathrm{J} 2000)\end{array}$ & $\begin{array}{l}\text { Period } \\
(\mathrm{d})\end{array}$ & $\begin{array}{l}V \\
(\mathrm{mag})\end{array}$ & $\begin{array}{l}I_{C} \\
(\mathrm{mag})\end{array}$ & $\begin{array}{l}K_{C I T} \\
(\mathrm{mag})\end{array}$ & $\mathrm{SNR}$ & $\begin{array}{c}\mathrm{RV} \\
\left(\mathrm{km} \mathrm{s}^{-1}\right)\end{array}$ & $\begin{array}{c}v \sin i \\
\left(\mathrm{~km} \mathrm{~s}^{-1}\right)\end{array}$ & $\begin{array}{l}\text { Run } \\
\text { Nos. }\end{array}$ \\
\hline N2516-1-1-1470 & 75708.92 & -612918.6 & 8.803 & 17.60 & 15.45 & 13.47 & 26 & $-0.56 \pm 0.47$ & $<8.00$ & 9 \\
N2516-1-1-1667 & 75716.58 & -613138.7 & 1.347 & 15.82 & 14.50 & 12.72 & 44 & $0.16 \pm 0.46$ & $21.98 \pm 1.91$ & 9 \\
N2516-1-1-1880 & 75724.78 & -612556.6 & - & 18.92 & 16.46 & 14.24 & 14 & $-2.82 \pm 0.73$ & $<8.00$ & $9 *$
\end{tabular}

Table 4. Chromospheric activity indices and related data for targets in NGC 2516. The identifier, period and light curve amplitudes are from IHA07. Luminosity, convective turnover time and Rossby number are calculated as described in Section 2.4 (using $(M-m)_{0}=7.93, A_{V}=0.37$, and $A_{I}=0.20$ ).

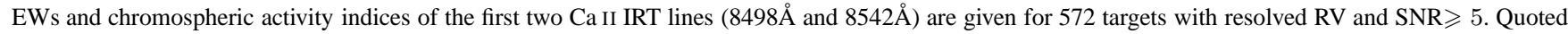
uncertainties in EW and activity indices do not include possible systematic errors (see JJ10). Masses are estimated from the models of Baraffe et al. (1998) and the absolute $I$ magnitude. The full table is available on Blackwell Synergy as Supplementary Material to the on-line version of this paper.

\begin{tabular}{lcccccccccc}
\hline Identifier & $\begin{array}{c}\log _{10} \\
L / L_{\odot}\end{array}$ & $\begin{array}{c}\text { Period } \\
\text { (days) }\end{array}$ & $\begin{array}{c}\text { sine } \\
\text { amplitude } \\
\text { (mag.) }\end{array}$ & $\begin{array}{c}\text { Turnover } \\
\text { time } \\
\text { (days) }\end{array}$ & $\begin{array}{c}\log _{10} \\
\text { Rossby } \\
\text { No. }\end{array}$ & $\begin{array}{c}\mathrm{EW}_{8498} \\
(\AA)\end{array}$ & $\begin{array}{c}\mathrm{EW}_{8542} \\
(\AA)\end{array}$ & $\begin{array}{c}\log _{10} \\
\mathrm{R}^{\prime}{ }_{C a 8498}\end{array}$ & $\begin{array}{c}\log _{10} \\
\mathrm{R}^{\prime}{ }_{C a 8542}\end{array}$ & $\begin{array}{c}\mathrm{Mass} \\
M_{\odot}\end{array}$ \\
\hline $\mathrm{N} 2516-1-1-1470$ & -1.23 & 8.803 & .016 & 52 & -0.77 & $0.45 \pm 0.02$ & $0.50 \pm 0.03$ & $-4.47 \pm .02$ & $-4.42 \pm .02$ & 0.57 \\
N2516-1-1-1667 & -0.88 & 1.347 & .010 & 35 & -1.41 & $0.42 \pm 0.01$ & $0.59 \pm 0.01$ & $-4.49 \pm .01$ & $-4.34 \pm .01$ & 0.70 \\
N2516-1-1-1880 & -1.62 & - & - & 81 & - & $0.07 \pm 0.04$ & $0.01 \pm 0.04$ & $-5.27 \pm .03$ & $-6.18 \pm .04$ & 0.43
\end{tabular}

$\left.4.75-M_{V}-B C_{V}\right)$ is calculated using a distance modulus of 7.93 (Terndrup et al. 2002) and bolometric corrections, $B C_{V}$, interpolated as a function of $(V-I)_{0}$ using the tables of Kenyon and Hartmann (1995). Colours were corrected assuming a uniform reddening of $E(B-V)=0.12$ (Terndrup et al. 2002), ratios of selective to total extinction $A_{V} / E(B-V)=3.1, A_{K} / E(V-K)=0.13$ (Rieke \& Lebofsky 1985) and $E(V-I)=0.16$ (Jeffries et al. 2001). A stellar mass is estimated by interpolating the models of Baraffe et al. (1998) using the absolute $I$ magnitudes.

Rossby numbers are given for stars with measured rotation periods, $P$, calculated as $N_{R}=P / \tau_{c}$, where $\tau_{c}$ is the convective turnover time. Rossby numbers are widely used to characterise the combined effects of rotation and spectral type on the activity levels of $\mathrm{F}-\mathrm{K}$ stars. Their use for M-dwarfs is less clear since the widelyused semi-empirical formula of Noyes et al.(1984) giving $\log \tau_{c}$ as a function of $B-V$ is unconstrained for M-dwarfs. An alternative approach is followed here where the value of $\tau_{c}$ is chosen such that chromospheric and coronal activity indicators satisfy a single scaling law with Rossby number irrespective of stellar mass. Pizzolato et al. (2003) noted that the mass dependence of a turnover time defined in this way is closely reproduced by assuming $\tau_{c} \propto L_{\mathrm{bol}}^{-1 / 2}$. Anchoring the turnover time of a solar type star as $\log \tau_{c}=1.1$, this gives the following expression;

$\log N_{R}=\log P-1.1+0.5 \log \left(L_{\mathrm{bol}} / L_{\odot}\right)$.

A few of the coolest stars in our sample (spectral type M4 and cooler, $I \gtrsim 18$ ) may be fully convective. The meaning of a turnover time in these objects is ill-defined. We apply equation 4 to these stars too, since others have shown that this parameterisation describes the behaviour of rotation and activity in stars at or just beyond the fully-convective boundary quite well. They follow the same relationship between activity and Rossby number as higher mass stars and their magnetic activity saturates at a similar value of $N_{R} \simeq 0.1$ (Kiraga \& Stepien 2007; Jeffries et al. 2011).

\section{RESULTS}

\subsection{Membership}

Targets were identified as potential cluster members by IHA07 from their position in the $V / V-I$ CMD. We make a further selection based on RV relative to the mean RV of cluster members in order to establish a more secure membership list. Chromospheric activity and rotation were not used as indicators of membership since we wish to study the distributions of these in cluster members. Fig. 2 shows an RV histogram; the RVs of stars with measured periods (shown as filled bars) are tightly bunched within a few $\mathrm{km} \mathrm{s}^{-1}$, indicating that the majority of these are cluster members. Stars without measured periods (shown as open bars) show a clear peak too but a much broader spread, presumably due to the presence of a proportion of contaminating older field stars.

Cluster members were iteratively defined as those stars with $\mathrm{RV}$ less than $2 \sigma_{e}$ from their mean RV, where $\sigma_{e}$ is the effective velocity dispersion due to the combined effects of RV uncertainties and the true velocity dispersion of the cluster. The true velocity dispersion of the cluster was estimated from the standard deviation of relative RV for targets with $v \sin i<20 \mathrm{~km} \mathrm{~s}^{-1}$, clipped at $\pm 5 \mathrm{~km} \mathrm{~s}^{-1}$, giving a result of $0.66 \pm 0.17 \mathrm{~km} \mathrm{~s}^{-1}$. Hence, from equation 1

$\sigma_{e}=\sqrt{\left(9.2+0.013(v \sin i)^{2}\right)^{2} / \mathrm{SNR}^{2}+0.72^{2}} \mathrm{~km} \mathrm{~s}^{-1}$.

Using equation 5 yields 354 probable cluster members, of which 210 have reported periods (see Fig. 2). A few fast rotating stars with correspondingly large RV uncertainties were included as members even though their RVs are some distance from the mean in absolute terms. These are still very probable members since fast rotators are comparatively rare amongst late-K to mid-M dwarf field stars (Delfosse et al. 1998). To estimate the maximum number of background stars falsely classified as members, the average number of targets in $\pm 2 \sigma_{e}$ bins centered at $\pm 10 \mathrm{~km} \mathrm{~s}^{-1}$ from the cluster mean was counted. This indicates that of stars identified as mem- 
Table 5. A summary of target numbers and the fraction of cluster members with rotation periods as a function of $I$ magnitude

\begin{tabular}{lcccccc}
\hline & Set 1 & Set 2 & Set 3 & Set 4 & Set 5 & All sets \\
\hline$I$ magnitude & 14.8 to 15.5 & 15.5 to 16.2 & 16.2 to 16.8 & 16.8 to 17.5 & 17.5 to 18.2 & 14.8 to 18.2 \\
$M_{I}$ & 6.7 to 7.3 & 7.3 to 8.0 & 8.0 to 8.7 & 8.7 to 9.4 & 9.4 to 10.1 & 6.7 to 10.1 \\
All targets (a) & $80+(4)$ & $99+(2)$ & $129+(0)$ & $116+(1)$ & $87+(2)$ & $511+(9)$ \\
Targets with period & $38+(1)$ & $59+(0)$ & $59+(0)$ & $46+(0)$ & $25+(0)$ & $227+(1)$ \\
Percentage of targets with period (b) & $48 \%$ & $60 \%$ & $46 \%$ & $40 \%$ & $29 \%$ & $44 \%$ \\
Percentage Monitor survey with period & $36 \%$ & $42 \%$ & $36 \%$ & $32 \%$ & $24 \%$ & $33 \%$ \\
Targets which are members & $50+(2)$ & $60+(1)$ & $81+(0)$ & $73+(1)$ & $67+(1)$ & $331+(5)$ \\
Members with period & $33+(1)$ & $49+(0)$ & $54+(0)$ & $44+(0)$ & $24+(0)$ & $204+(1)$ \\
Members without period & $17+(1)$ & $11+(1)$ & $27+(0)$ & $29+(1)$ & $43+(1)$ & $127+(4)$ \\
Fraction members with period (c) & 0.55 & 0.69 & 0.57 & 0.52 & 0.30 & 0.50 \\
Fraction with $N_{R} \leqslant 0.1$ & 0.55 & 0.86 & 1.00 & 1.00 & 1.00 & 0.89 \\
\hline
\end{tabular}

(a) Number of targets with with SNR $\geqslant 5$ where the number in brackets counts targets with unresolved membership.

(b) Percentage calculated neglecting targets with unresolved membership.

(c) Fraction after correction is made for the bias in the number of targets with resolved period in the spectroscopic sample.
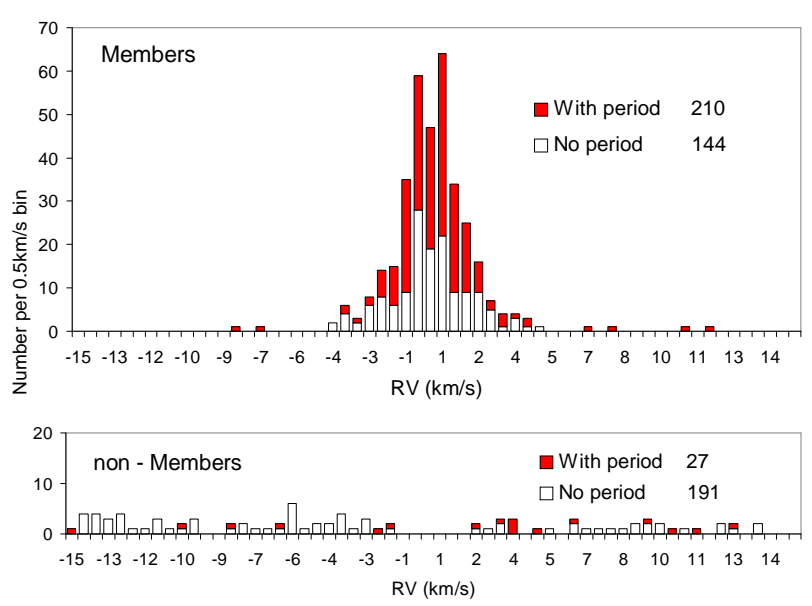

Figure 2. Radial velocity histograms for targets in NGC 2516 (relative to the mean RV of the cluster). The upper plot shows objects we classify as cluster members in $0.5 \mathrm{~km} \mathrm{~s}^{-1}$ bins. The lower plot shows targets classified as non-members. Note that $\sim 50$ percent of non members have RVs outside the range of this plot.

bers, only $3 / 210$ ( 1.5 per cent) with measured periods are likely to be non members whereas about 12/144 ( 8 per cent) without periods are possible non-members.

\subsection{Numbers of targets with and without measured periods}

In the following analysis the sample is restricted to 331 members with $14.8 \leqslant I \leqslant 18.2$ (204 with periods, 127 without), where there is sufficient target density (per unit magnitude) to make meaningful comparisons of stars with and without periods. Table 5 shows the numbers of stars with and without measured periods in five equally spaced bins in $I$. Also shown in brackets are the numbers of stars in each bin with SNR $\geqslant 5$ but unresolved RVs. (see Table 2).

The fraction of stars with measured periods in our sample was biased by our prior selection of a high proportion of targets with measured rotation periods. In contrast, the fraction of photometric candidates from IHA07 with measured periods is unbiased. The ratios of these proportions are used to scale the numbers of non members in our sample to obtain an unbiased estimate of the fraction of NGC 2516 members with measured periods. Whilst the average is about 50 per cent, this falls significantly for Bin 5 containing cooler stars. Finally, Table 5 shows the fraction of targets in each bin with measured periods that also have Rossby numbers $\leqslant 0.1$. These are expected to show saturated chromospheric activity (see JJ10). A significant proportion (45 per cent) of the stars in Bin 1 are unsaturated. These show an average Ca II IRT activity index of about half the saturated level. For practical purposes all targets in the fainter (cooler) bins have saturated chromospheric activity levels.

\section{THE PROPERTIES OF LOW-MASS STARS WITH AND WITHOUT MEASURED PERIODS}

In this section we examine NGC 2516 members with $6.7 \leqslant M_{I} \leqslant$ 10.1 for evidence of differences in the photometric, chromospheric or rotational properties of stars with and without measured periods.

\subsection{Colour-magnitude diagrams}

Figure 5 shows $M_{V}$ versus $(V-I)_{0}$ and $M_{I}$ vs $(I-K)_{0}$ CMDs for targets in NGC 2516 with measured RV and SNR $\geqslant 5$. Approximate spectral types are indicated using colour to spectral type relations from Kenyon and Hartmann (1995). Cluster members with and without measured periods and RV non-members are indicated separately. Also shown are trend lines representing a third order polynomial fit to all cluster members together with $1 \sigma$ standard deviations and polynomials representing colour-magnitude relations for nearby field stars. The latter are fits to field star data from the on-line database of Reid (2002). Both plots show offsets between NGC 2516 members and the trend line for field stars. This is not surprising since the field stars are an older population with inhomogeneous metallicity. Of more interest are the very small differences in the offsets for stars with and without measured periods.

Stars with $6.7<M_{I}<10.1$ and measured periods have an offset of $-0.012 \pm 0.005 \mathrm{mag}$ in $(V-I)_{0}$ relative to the mean trend line, whereas stars without measured periods have an offset of $0.009 \pm 0.009 \mathrm{mag}$. In the $M_{I}$ versus $(I-K)_{0}$ CMD stars with measured period have an offset of $-0.006 \pm 0.008$ mag in $(I-K)_{0}$, whereas stars without measured periods have an offset of $-0.007 \pm 0.014 \mathrm{mag}$. Given that some small systematic differences between the two groups are expected, for example in the expected level of field star contamination in the two groups, these results do not indicate any difference in colour between the two groups. By 

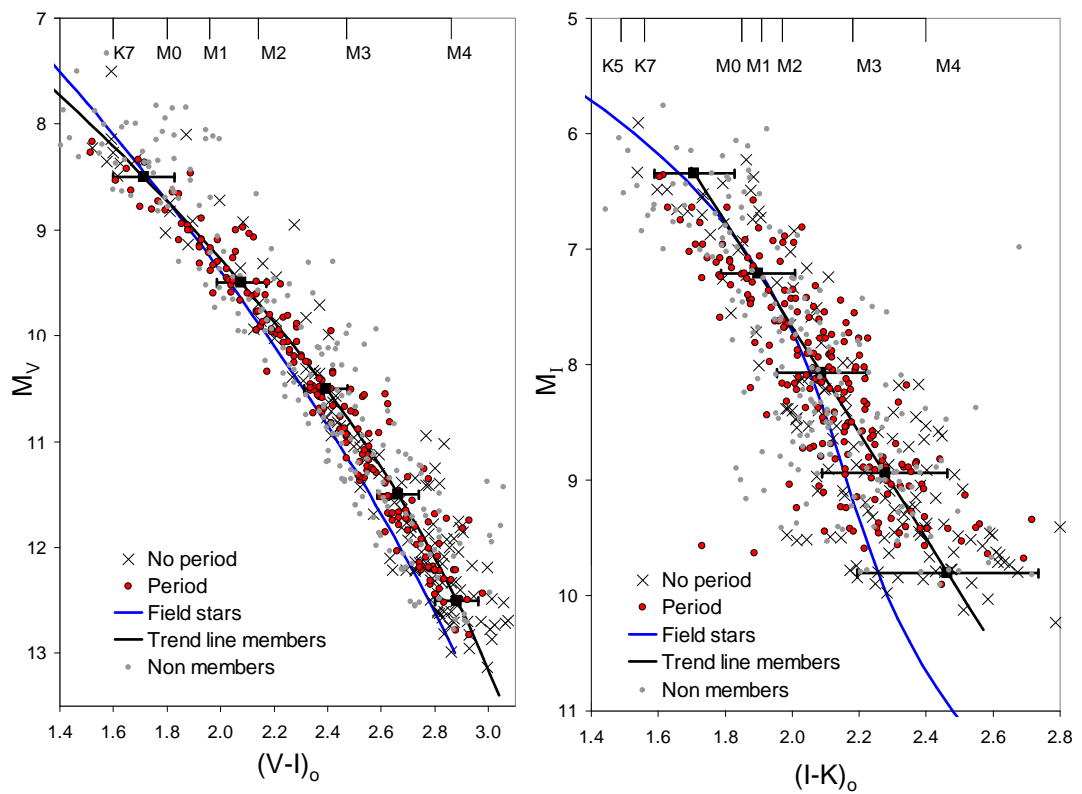

Figure 3. $M_{V}$ versus $(V-I)_{0}$ and $M_{I}$ vs $(I-K)_{0}$ colour magnitude diagrams for targets in NGC 2516 with SNR $\geqslant 5$ (using $(M-m)_{0}=7.93, A_{V}=0.37, A_{I}=0.20$ and $\left.A_{K}=0.04\right)$. Circles and crosses indicate targets confirmed as members (with and without periods). Dots are non-members (assuming the same distance modulus and reddening). Also shown are: (a) a trend line to all cluster members together with $1 \sigma$ standard deviations; (b) a polynomial representing the mean absolute magnitude of nearby field stars as a function of colour (see section 4.1).

inference, there is little or no difference in effective temperature as a function of luminosity between stars with and without measured periods whereas one would be expected if they had differing levels of starspot coverage (Jackson et al. 2009).

\subsection{Chromospheric activity}

Figure 4 shows the CaII IRT activity indices for members of NGC 2516 with and without measured periods as a function of absolute $I$. Ca II IRT activity was compared between the two data sets by selecting cluster members with $6.7<M_{I}<10.1$ and Ca II IRT activity index $>10^{-5}$ and measuring the offset of the measured Ca II IRT activity index from a linear regression line as a function of $I$ for the whole dataset (see Fig. 4). The mean offsets, $0.010 \pm 0.008$ dex for targets with periods and $0.018 \pm 0.012 \mathrm{dex}$ for members without periods, indicate no resolvable difference in their mean levels of chromospheric activity.

\subsection{Equatorial velocity}

The distribution of $v \sin i$ for M-dwarfs in NGC 2516 is similar to that seen in other clusters of similar age (e.g. the Pleiades; Queloz et al. 1998, Terndrup et al. 2000). There is a wide range of rotation rates, with significant populations of more slowly rotating stars ( $v \sin i$ unresolved), a central band where the number density falls with increasing $v \sin i$ with a scale length of about $30 \mathrm{~km} \mathrm{~s}^{-1}$ and a tail of fast rotators with $50<v \sin i<150 \mathrm{~km} \mathrm{~s}^{-1}$.

The top left plot of Fig. 5 shows cumulative probability distributions of $v \sin i$ for stars with $v \sin i \geqslant 8 \mathrm{~km} \mathrm{~s}^{-1}$, with and without measured periods. The distributions appear similar although targets with measured periods have an excess tail of fast rotators. A 2-sided Kolmogorov-Smirnov test yields a 75\% probability that the individual distributions are consistent with being drawn from an

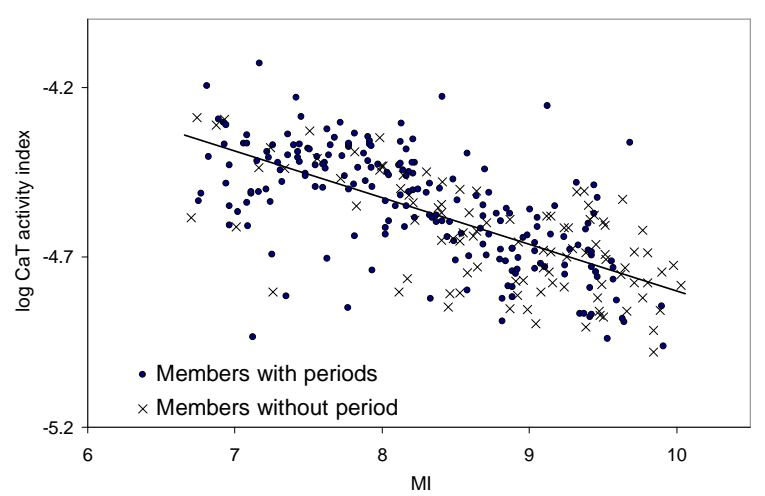

Figure 4. Summed chromospheric activity indices for the first two lines of the Ca II IRT versus absolute $I$ for members of NGC 2516 with spectral $\mathrm{SNR} \geqslant 5$ and a mean activity index $>10^{-5}$. Also shown is a regression line for all members with $6.7<M_{I}<10.1$.

identical distribution. The fractions of stars with $v \sin i<8 \mathrm{~km} \mathrm{~s}^{-1}$ (51/204 versus 26/127) are also indistinguishable.

Fig. 5 provides more detailed comparisons of the $v \sin i$ distributions for members with and without periods as a function of absolute $I$. The four bins containing higher mass stars have between a 29 and $>99$ per cent probability that the individual distributions are consistent with being drawn from the same parent distribution. However, in Bin 5 the proportion of stars with measured periods is reduced and the median $v \sin i$ is $7 \mathrm{~km} \mathrm{~s}^{-1}$ higher for stars with measured periods than for those without periods. This could signify fundamental differences in the velocity distributions of cooler stars with and without the spotted photospheres required to modulate light curves, but equally it could reflect an anticipated measurement bias because it becomes increasingly difficult to measure 

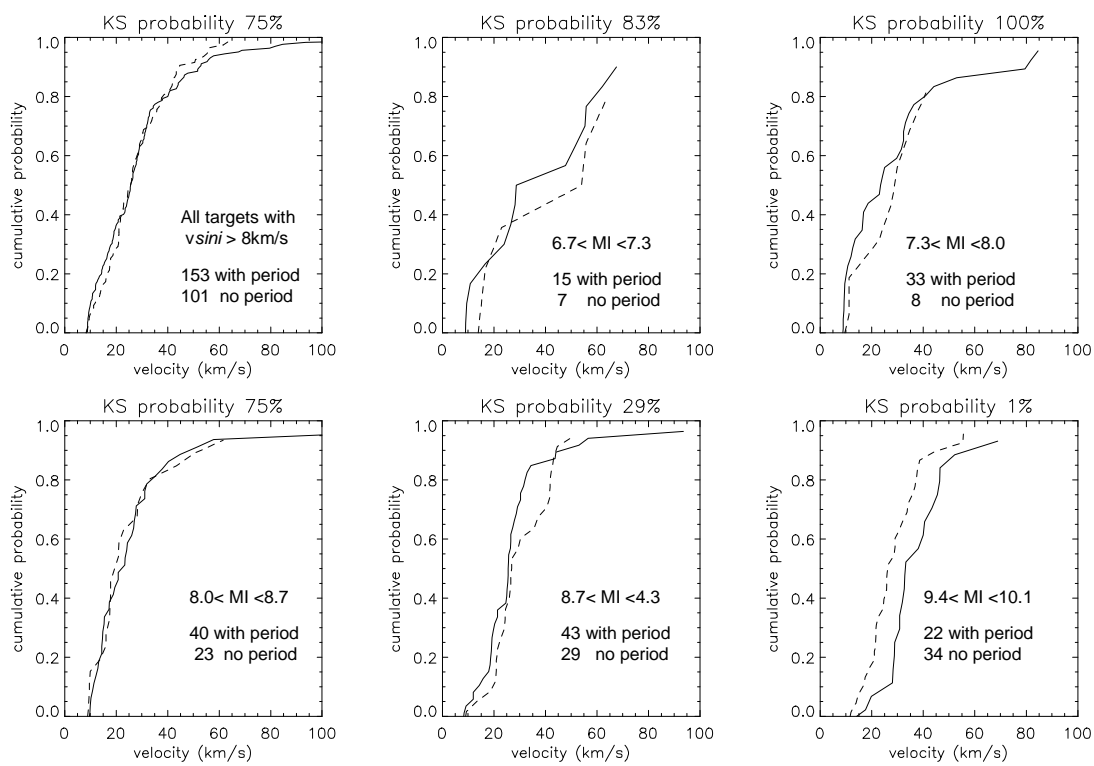

Figure 5. A comparison of the cumulative distribution of $v \sin i$ for members of NGC 2516 with and without measured periods. The solid line shows stars with periods the dashed line shows those without. The top left plot compares all targets with $v \sin i \geqslant 8 \mathrm{~km} \mathrm{~s}^{-1}$. The remaining plots compare distributions in 5 equal bins of $I$ magnitude (see Table 5). Probabilities that data sets are consistent with being drawn from a common distribution are shown above each plot.

longer periods for fainter stars, due to their larger photometric uncertainties (see Fig. 5 of IHA07).

\subsection{A hypothesis based on the distribution of starspots}

If there are no significant differences in the colours, magnetic activity and (for all but the faintest stars) no difference in rotation speed for stars with and without measured periods, then what is causing the apparent difference in their light curves? An obvious possibility is that there is some difference in observational sensitivity between the two groups. Fig. 6 shows the expected light curve amplitude uncertainty as a function of $I$. The majority of targets were observed 270 to 320 times. Stars with and without measured periods show similar levels of uncertainty. Thus accuracy of the observations is not a discriminating factor between the two groups. Seven stars were observed less than 270 times and show anomalously high uncertainties (see Table 6). None have measured periods, but 6 of the 7 are bright stars where only a small number of points were lost due to saturation. For these stars, although the uncertainty is increased above the norm, it could still be low enough to detect significant light curve modulation. One target (N2516-3-5-3994) was at the extreme edge of a detector and was rarely sampled. For simplicity we decided to retain all these stars in our analysis. As a check, the comparisons of colour, chromospheric activity and the distribution of $v \sin i$ for stars with and without measured periods were repeated without these 7 stars. The results were unchanged.

A second possible cause of observational bias is contamination of light curves by adjacent stars. This was checked by comparing the frequency with which a deblending algorithm was triggered during the photometric analysis. The criterion for this was that isophotes of any adjacent stars (averaged over 20 images) overlapped beyond a detection threshold of 3 sigma above sky (Irwin 1985). The algorithm was triggered for 22 per cent of stars with measured periods compared to 28 per cent of stars without periods.
This difference is less than the Poisson uncertainty for samples of 204 and 127 stars, so this appears not to be a significant factor preventing detection of periods in one group.

Having ruled out observational bias we can consider the physical properties of stars in the two groups. Stars with measured periods could show increased levels of spot coverage due to increased levels of magnetic activity. The evidence is against this since the two groups have indistinguishable levels of chromospheric (Ca II IRT) activity and are not displaced with respect to each other in CMDs. The similar distributions of $v \sin i$ also suggest similar levels of magnetic activity. It is possible that there is no relationship between levels of chromospheric activity and spot coverage, in which case the similarity of chromospheric activity levels has little significance, but in our view this is unlikely. The flux of equipartion magnetic field in M-dwarfs appears correlated with both rotation and magnetic activity assessed with chromospheric and coronal indicators (Reiners, Basri \& Browning 2009).

A second possibility is that stars without measured periods are slower rotators and their rotational periods are too long to have been measured by IHA07. This cannot be true since 80 per cent of members without periods have $v \sin i \geqslant 8 \mathrm{~km} \mathrm{~s}^{-1}$. There could be a difference in the period distribution of the slower rotators (with $v \sin i<8 \mathrm{~km} \mathrm{~s}^{-1}$ ). However even this is not feasible since the Monitor Survey of NGC 2516 claimed completeness $>75$ per cent for periods $\leqslant 20$ days. Empirical activity-rotation relationships show that saturated levels of magnetic activity in early- and midM dwarfs occur for rotation periods shorter than 8-15 days and decrease rapidly for longer periods (e.g. Kiraga \& Stepien 2007; Jeffries et al. 2011). Hence, if there were a much higher proportion of long period stars ( $>20$ days) in the group without periods this would be indicated by reduced chromospheric activity levels.

A third possibility is that stochastic variations associated with flaring activity might mask starspot modulation. This seems unlikely because the rms variation in the light curves of those stars without detected periods is significantly lower on average than 


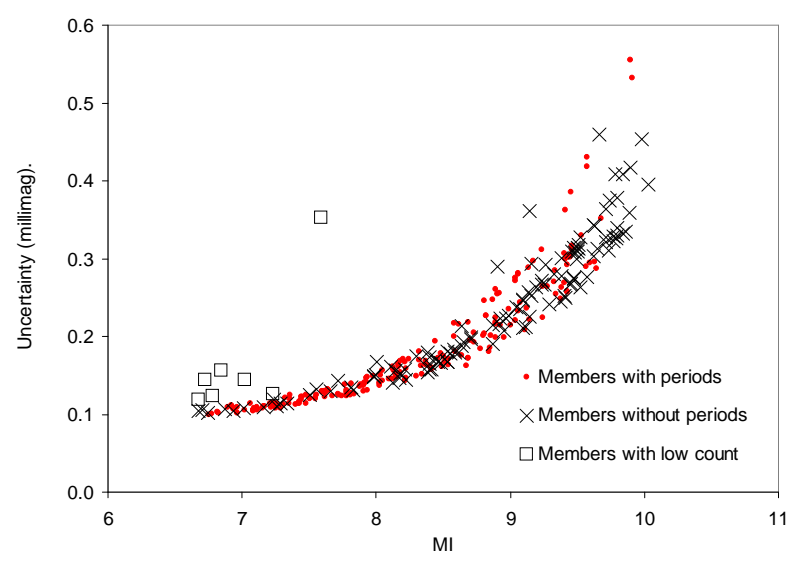

Figure 6. Predicted uncertainty in light curve amplitude as a function of $I$ $\left(6.7<M_{I}<10.1\right)$ for members of NGC 2516 with and without periods. Circles and crosses show results for cluster members with $>270$ photometric points defining their light curves. Squares show targets with lower numbers of points in their light curves.

those in which modulation was detected (see Table 7). That is, the light curves of stars without detected periods are flatter, not noisier.

A final possibility is that the stars without measured periods are predominantly viewed at low inclination angles, reducing their light curve amplitudes. There are two arguments against this hypothesis.

(i) The inclination below which spot modulation becomes undetectable would have to be unreasonably high. In a simple model where half of stars show no modulation because of their inclination, the critical angle would be $i \simeq 60^{\circ}$. Because the number density of objects with inclination $i$ varies as $\sin i$, most stars with undetected periods would have $40^{\circ}<i<60^{\circ}$ but it seems unlikely this would be low enough to prevent spot modulation. There are many examples in the literature where spot modulation is seen in stars with $i<60^{\circ}$ (e.g LO Peg with $i=50^{\circ}$, Jeffries et al. 1994; HR 1099 with $i=38^{\circ}$, Lanza et al. 2006; PW And with $i=46^{\circ}$, Strassmeier and Rice 2006).

(ii) A systematically lower inclination would lead to very different $v \sin i$ distributions in stars with and without periods. Making the reasonable assumption that rotational period and inclination are unrelated, then for a critical angle of $60^{\circ}$ the ratio of the mean value of $v \sin i$ for stars without periods to those with periods would be,

$\int_{0}^{\pi / 3} \sin ^{2} i d i / \int_{\pi / 3}^{\pi / 2} \sin ^{2} i d i=0.64$

The similar $v \sin i$ distributions in Fig. 5 suggest this is not the case. To test the significance of this consider the $v \sin i$ distribution of the 204 stars with measured periods, where 50 per cent have $v \sin i>18.9 \mathrm{~km} \mathrm{~s}^{-1}$. If the average $\sin i$ were reduced by a factor 0.64 , then for the same velocity distribution only 27 per cent of stars would have $v \sin i$ above this value. Hence among the 127 stars without periods, we would expect only 34 to have $v \sin i>18.9 \mathrm{~km} \mathrm{~s}^{-1}$, compared with the 77 observed. This discrepancy means that the possibility that the difference between stars with and without measured periods is determined solely by their inclination can be rejected with $>99.9$ percent confidence according to a two-tailed chi-squared test.
Table 6. Targets with less likelihood of measuring a rotation period because there were fewer photometric points in their light curves. None of these targets have measured periods.

\begin{tabular}{lccc}
\hline Name & $\mathrm{M}_{I}$ & $\operatorname{vsin} i\left(\mathrm{~km} \mathrm{~s}^{-1}\right)$ & Count \\
\hline N2516-1-3-1740 & 6.68 & - & 234 \\
N2516-1-8-3126 & 6.73 & 13.1 & 155 \\
N2516-3-5-3341 & 6.79 & - & 211 \\
N2516-1-4-470 & 6.85 & - & 141 \\
N2516-1-4-401 & 7.03 & 23.0 & 181 \\
N2516-1-4-673 & 7.24 & - & 244 \\
N2516-3-5-3994 & 7.60 & 27.7 & 38 \\
\hline
\end{tabular}

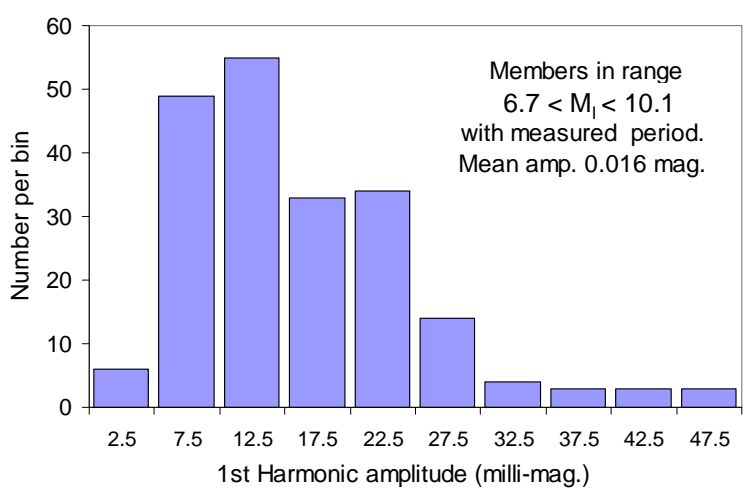

Figure 7. Histogram of light curve modulation amplitudes for targets with resolved periods and identified as members of NGC 2516.

Rejection of all these possibilities favours hypotheses that do not require any fundamental difference between the two groups of stars. If stars with and without measured periods have similar distributions of rotation rate, and similar levels magnetic activity and spot coverage then the principle difference between stars with and without measured periods must be a chance combination of:

(i) the distribution of spots on the stellar surface which affects the "true" light curve amplitude. This could be a random effect of starspot placement or a systematic effect where, for example, stars with measured periods have a higher proportion of equatorial spots.

(ii) the effects of inclination and photometric uncertainties modulating the "true" light curve and producing the measured light curve from which a period is or is not measured.

\subsection{The distribution of light curve modulation amplitudes}

The method used by IHA07 to detect periodic modulation and determine the periods and amplitudes was described in detail by Irwin et al. (2006). Sine curves were fitted to data over a grid of frequencies. Periodic light curves were selected on the basis of a reduced $\chi^{2}$ test, followed by visual inspection of the folded light curve. For stars with resolved periods IHA07 reported the first harmonic amplitude of the best fitting sine wave. Results for our list of target stars are reproduced in Table 4. Fig. 7 shows a histogram of light curve amplitudes for the members of NGC 2516 with measured periods. The most striking feature is how small the amplitudes are; the mean is 0.016 mag. and all are below 0.05 mag. 


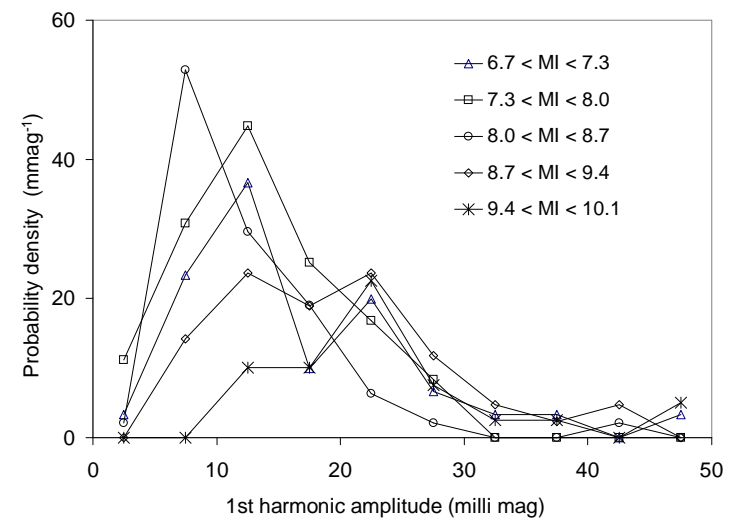

Figure 8. Probability density of light curve modulation amplitudes for members of NGC 2516 split according to their absolute $I$ magnitudes. The neasured frequencies are normalised according to the total number of targets in each set (see Table 5) such that the probability density summed over all amplitudes gives the fraction of targets in a set with measured period.

Using the fraction of stars with measured periods from Table 5, the light curve amplitude data are presented in Fig. 8 as probability distributions for the same five $I$ magnitude bins considered in previous sections. The properties of these distributions are summarised in Table 7. Summing the probability densities for each dataset gives the total probability of measuring a period in each bin. The distribution functions for each bin are similar, rising with harmonic amplitude before decaying to zero for amplitudes $\geqslant 0.05 \mathrm{mag}$. The peak in probability density shifts to higher amplitudes for the fainter stars of bins 4 and 5 . This could be a real effect or, more likely, reflects the increasing difficulty in resolving periodicity for fainter stars, as photometric uncertainties become comparable with light curve amplitudes.

\section{DISCUSSION}

A previous analysis of a subset of these data used $v \sin i$ and rotational period to estimate projected radii $(R \sin i)$ for 210 fast rotating members of NGC 2516 and determine mean stellar radii (Jackson et al. 2009). Stars with $M_{I}>7.3$ have measured radii much higher than expected from evolutionary models and much higher than inactive field stars of similar luminosity. The larger radii and cooler effective temperatures are consistent with up to $\sim 50$ percent of the stellar surface being covered by dark starspots (see Fig. 3 of Jackson et al. 2009). Despite this large starspot coverage these stars show only low levels of variation in luminosity with rotation (see Table 7 and Fig. 8), indicating that only a small fraction of the darkened surface is modulating the light curve. In quantitative terms, a single dark spot covering 1 per cent of the star's total surface is sufficient to cause the typical light curve amplitudes of $0.02 \mathrm{mag}$. This suggests as little as $\sim 2$ per cent of the darkened area is responsible for the light curve amplitude.

A possible explanation could be that spots are concentrated at uneclipsed polar latitudes. This effect would have to be extreme; up to 98 per cent of the darkened surface would need to be in axisymmetric spots or permanently visible (or invisible) at the poles. Young et al. (1990) proposed high latitude activity to explain the light curves of a nearby, rapidly rotating M0 dwarf, HK Aqr. However, more recent Doppler imaging measurements of this and a sec- ond rapidly rotating M1.5 dwarf (EY Dra) by Barnes \& Collier Cameron (2001) showed no evidence for polar spots. The majority of spots appeared at low latitudes on HK Aqr. EY Dra showed spots at all latitudes. Evidence for spot concentration, polar or equatorial, remains sparse for late $\mathrm{K}$ and early $\mathrm{M}$-dwarfs with few measured in any detail (e.g. Barnes et al. 2005). There is no evidence of polar spots extending down to the latitudes of $30^{\circ}$ that would be required to cover 50 per cent of the stellar surface.

An alternative explanation is that a large number of small starspots are randomly distributed over the stellar surface. As the star rotates the light curve would correspond to the average luminosity of many small areas of varying luminosity. If these areas are sufficiently small then the variation in the stellar luminosity would be a small fraction of the change in luminosity from the immaculate surface to the starspot. For example, consider a star with 50 per cent surface coverage by 5000 randomly placed dark starspots of order $2^{\circ}$ in diameter. At any time 2500 spots would be visible to the observer and the variation of luminosity with rotation would be of the order $1 / \sqrt{2500}$ - about $\sim 0.02$ mag as found in our sample (see Table 7).

The same hyothesis also explains why some stars have measured periods and some do not. There is no difference between the two groups of stars it is simply a matter of chance. In some cases the variations in luminosity over a cycle contain a distinct first harmonic component producing a measured period. In other cases the first harmonic component is so low it cannot be resolved from the background of higher order harmonics in the light curve.

The random spot distribution hypothesis need not be the sole explanation for low light curve amplitudes. There could effectively be two starspot distributions, one axisymmetric/polar and one randomly distributed over the stellar surface. Both distributions would reduce $T_{\text {eff }}$ and increase the radius of active stars (at a given luminosity) but only the randomly distributed spots could contribute to the measured light curve modulation. However any axisymmetric/polar component would have to be very large to significantly change the starspot size required to produce the measured light curve amplitudes, since this only varies as the square root of filling factor. If half the spot coverage were permanently axisymmetric, this would only increase the estimated size for the randomly distributed spots from $\sim 2^{\circ}$ to $\sim 3^{\circ}$. A further possibility is the presence of bright plages contributing an additional source of variation in surface luminosity. However, it is reasonable to suppose that were plages present in significant numbers then their scale length would also need to be comparable to that of the dark starspots, otherwise they would increase the light curve amplitudes.

Our simplified calculation neglects the important effects of inclination, limb darkening and how random intensity variations combine to produce a measurable light curve amplitude. As such it probably overestimates the number of spots and underestimates their size. Even so it serves to demonstrate that the observed high spot filling factor (up to $\sim 50$ per cent) and low levels of light curve amplitudes ( $\sim 0.02 \mathrm{mag}$ ) of fast rotating M-dwarfs in NGC 2516 are consistent with their surface being covered by a large number of randomly distributed starspots of order $2^{\circ}$ in diameter.

Small starspots can also explain why the total magnetic flux derived from Stokes V measurements of active M-dwarfs is only 615 per cent of the total magnetic flux implied by Stokes I measurements, indicating that the magnetic flux on M-dwarfs is stored in small scale components (Reiners \& Basri 2009). The Stokes I component is found from Zeeman broadening of magnetically sensitive lines produced by magnetic field along the line of sight, irrespective of polarity (Johns-Krull and Valentini 1996). As such, it is sensitive 
Table 7. Average characteristics of the $I$ band light curves of low-mass members of NGC 2516 as a function of absolute $I$ magnitude

\begin{tabular}{lcccccc}
\hline$M_{I}$ & 6.7 to 7.3 & 7.3 to 8.0 & 8.0 to 8.7 & 8.7 to 9.4 & 9.38 to 10.06 & 6.66 to 10.06 \\
\hline Light curve rms for members with period (mag) & 0.010 & 0.008 & 0.008 & 0.012 & 0.015 & 0.010 \\
Light curve rms for members without period (mag) & 0.007 & 0.003 & 0.005 & 0.008 & 0.006 & 0.008 \\
Mean first harmonic of light curve (mag o-p) (a) & 0.017 & 0.014 & 0.012 & 0.020 & 0.024 & 0.016 \\
Maximum light curve amplitude (mag o-p) (a) & 0.049 & 0.030 & 0.041 & 0.045 & 0.049 & 0.049 \\
\hline
\end{tabular}

(a) Stars with measured periods

to the average magnetic field and total filling factor of starspots, but gives no indication of their scale length or distribution. Conversely, the circularly polarized component of magnetic field, the Stokes V component, detects total field along the line of sight taking account of its polarity. Periodic modulation of the Zeeman signature due to variations in projected velocity with time are used to determine the surface distribution of magnetic field, or more precisely, to estimate the magnetic field distribution in terms of the spherical harmonics of poloidal and toroidal fields that best match the data (Donati et al. 2006). The surface detail that can be resolved depends on the limit of the spherical harmonic expansion. The resolution increases with the rotational speed of the star but is typically 8 terms for moderate rotators, limiting resolution to magnetic features of size $>7^{\circ}$ $(180 / 8 \pi)$. Morin et al. (2008) suggest a higher resolution is possible (70 elements around the equatorial ring) but used the same limit of 8 spherical harmonics for their measurements of mid-M dwarfs.

If a significant proportion of the surface of active M-dwarfs is covered by starspots of scale length $\sim 2^{\circ}$, then these will contribute strongly to the total Stokes I signature but will not be resolved by Zeeman Doppler imaging and will not contribute significantly to the Stokes V component, although there would be some small signature at high filling factors whenever random groupings of small starspots form larger features on the stellar surface. This explanation depends critically on the actual scale length of starspots being significantly less than the current angular resolution of Zeeman Doppler imaging.

\section{SUMMARY}

In this paper we have presented intermediate resolution spectroscopic observations for two large samples of low-mass stars in the young cluster NGC 2516. The two samples are distinguished by whether previous photometric observations were able to discern significant rotational modulation in their $I$-band light curves. Cluster membership has been confirmed using a common radial velocity criterion and after correcting for target selection biases, we find that approximately 50 per cent of low-mass NGC 2516 members fall into each category.

The projected equatorial velocities, positions in colourmagnitude diagrams and levels of chromospheric activity for the two samples have been compared and show no significant differences. The vast majority of our sample show very high levels of magnetic activity and fast rotation rates, whether they have measured rotation periods or not. Neither are there any differences in observation cadence or sensitivity that would explain why a large fraction of stars do not show spot-modulated light curves. Explanations involving differences in magnetic activity, rotation rate or spin-axis inclination are ruled out and we argue that the overall spot coverage is likely to be similar in both sets of stars. Instead we propose that the lack of spot modulation in many stars and the low amplitude (0.01-0.02 mag) modulation seen even in those stars which do have measured periods, can be explained if the spot coverage consists of large numbers of randomly placed spots of diameter $\sim 2^{\circ}$. Such spots would be impossible to resolve using current Doppler imaging and Zeeman Doppler imaging techniques and may explain why only a small fraction of magnetic flux is detected in the large scale structures probed by Stokes V measurements.

The next step in testing this hypothesis will be to develop a quantitative model examining spot coverage as a function of mass or spectral-type and attempt to simultaneously explain the detailed distribution of light curve modulation amplitudes and the fraction of stars with and without measured rotational modulation.

\section{ACKNOWLEDGEMENTS}

Based on observations collected at the European Southern Observatory, Paranal, Chile through observing programs 380.D-0479 and 266.D-5655. RJJ acknowledges receipt of a Wingate scholarship. The authors would like to thank Dr J Irwin and Dr S Aigrain for their help with target selection for this project and for providing additional, previously unpublished, data relating to their photometric light curves.

\section{REFERENCES}

Agüeros M. A., Covey K. R., Lemonias J. J., Law N. M., Kraus A., Batalha N., Bloom J. S., Cenko S. B., Kasliwal M. M., Kulkarni S. R., Nugent P. E., Ofek E. O., Poznanski D., Quimby R. M., 2011, ApJ, 740, 110

Aigrain S., Hodgkin S., Irwin J., Hebb L., Irwin M., Favata F., Moraux E., Pont F., 2007, MNRAS, 375, 29

Bagnulo S., Jehin E., Ledoux C., Cabanac R., Melo C., Gilmozzi R., The ESO Paranal Science Operations Team 2003, The Messenger, 114,10

Baraffe I., Chabrier G., Allard F., Hauschildt P. H., 1998, A\&A, 337,403

Barnes J. R., Collier Cameron A., 2001, MNRAS, 326, 950

Barnes J. R., Collier Cameron A., Donati J., James D. J., Marsden S. C., Mengel M., Petit P., 2005, in F. Favata, G. A. J. Hussain, \& B. Battrick ed., 13th Cambridge Workshop on Cool Stars, Stellar Systems and the Sun. ESA Special Publication Vol. 560 of ESA Special Publication, The decrease in differential rotation with decreasing stellar mass in a sample of young rapidly rotating main sequence stars. pp 95-+

Barnes J. R., Jeffers S. V., Jones H. R. A., 2011, MNRAS, 412, 1599

Carpenter J. M., 2001, AJ, 121, 2851

Chabrier G., Gallardo J., Baraffe I., 2007, A\&A, 472, L17

Collier-Cameron A., Unruh Y. C., 1994, MNRAS, 269, 814 
Cutri, R. M. et al. 2003, Technical report, Explanatory supplement to the 2MASS All Sky data release. http://www.ipac.caltech.edu/2mass/

Czesla S., Huber K. F., Wolter U., Schröter S., Schmitt J. H. M. M., 2009, A\&A, 505, 1277

Dachs J., Kabus H., 1989, A\&AS, 78, 25

Delfosse X., Forveille T., Perrier C., Mayor M., 1998, A\&A, 331, 581

Delorme P., Collier Cameron A., Hebb L., Rostron J., Lister T. A., Norton A. J., Pollacco D., West R. G., 2011, MNRAS, 413, 2218 Donati J., Howarth I. D., Jardine M. M., Petit P., Catala C., Landstreet J. D., Bouret J., Alecian E., Barnes J. R., Forveille T., Paletou F., Manset N., 2006, MNRAS, 370, 629

Donati J.-F., Semel M., Carter B. D., Rees D. E., Collier Cameron A., 1997, MNRAS, 291, 658

Eaton J. A., Hall D. S., 1979, ApJ, 227, 907

Gray D. F., 1984, ApJ, 277, 640

Hall D. S., 1972, PASP, 84, 323

Hartman J. D., Bakos G. Á., Kovács G., Noyes R. W., 2010, MNRAS, 408, 475

Hodgkin S. T., Irwin J. M., Aigrain S., Hebb L., Moraux E., Irwin M. J., the Monitor collaboration 2006, Astronomische Nachrichten, 327, 9

Irwin J., Aigrain S., Bouvier J., Hebb L., Hodgkin S., Irwin M., Moraux E., 2009, MNRAS, 392, 1456

Irwin J., Aigrain S., Hodgkin S., Irwin M., Bouvier J., Clarke C., Hebb L., Moraux E., 2006, MNRAS, 370, 954

Irwin J., Bouvier J., 2009, in E. E. Mamajek, D. R. Soderblom, \& R. F. G. Wyse ed., IAU Symposium Vol. 258 of IAU Symposium, The rotational evolution of low-mass stars. pp 363-374

Irwin J., Hodgkin S., Aigrain S., Bouvier J., Hebb L., Moraux E., 2008, MNRAS, 383, 1588

Irwin J., Hodgkin S., Aigrain S., Hebb L., Bouvier J., Clarke C., Moraux E., Bramich D. M., 2007, MNRAS, 377, 741

Irwin M. J., 1985, in V. di Gesu, L. Scarsi, P. Crane, J. H. Friedman, \& S. Levialdi ed., Data Analysis in Astronomy Automatic Analysis of Crowded Fields. p. 439

Jackson R. J., Jeffries R. D., 2010, MNRAS, 407, 465

Jackson R. J., Jeffries R. D., Maxted P. F. L., 2009, MNRAS, 399, L89

James D. J., Barnes S. A., Meibom S., Lockwood G. W., Levine S. E., Deliyannis C., Platais I., Steinhauer A., Hurley B. K., 2010, A\&A, 515, A100+

Jeffries R. D., Byrne P. B., Doyle J. G., Anders G. J., James D. J., Lanzafame A. C., 1994, MNRAS, 270, 153

Jeffries R. D., Jackson R. J., Briggs K. R., Evans P. A., Pye J. P., 2011, MNRAS, 411, 2099

Jeffries R. D., James D. J., Thurston M. R., 1998, MNRAS, 300, 550

Jeffries R. D., Thurston M. R., Hambly N. C., 2001, A\&A, 375, 863

Johns-Krull C. M., Valenti J. A., 1996, in R. Pallavicini \& A. K. Dupree ed., Cool Stars, Stellar Systems, and the Sun Vol. 109 of Astronomical Society of the Pacific Conference Series, Detection of strong magnetic fields on M dwarfs. pp 609-+ Johns-Krull C. M., Valenti J. A., 2000, in Pallavicini R., Micela G., Sciortino S., eds, Stellar Clusters and Associations: Convection, Rotation, and Dynamos Vol. 198 of Astronomical Society of the Pacific Conference Series. pp 371-+

Kenyon S. J., Hartmann L., 1995, ApJS, 101, 117

Kiraga M., Stepien K., 2007, Acta Astronomica, 57, 149

Lanza A. F., Piluso N., Rodonò M., Messina S., Cutispoto G.,
2006, A\&A, 455, 595

Lyra W., Moitinho A., van der Bliek N. S., Alves J., 2006, A\&A, 453, 101

Makarov V. V., Beichman C. A., Catanzarite J. H., Fischer D. A., Lebreton J., Malbet F., Shao M., 2009, ApJ, 707, L73

Mallik S. V., 1994, A\&AS, 103, 279

Mallik S. V., 1997, A\&AS, 124, 359

Marcy G. W., 1982, PASP, 94, 989

Marsden S. C., Carter B. D., Donati J., 2009, MNRAS, 399, 888

Meibom S., Mathieu R. D., Stassun K. G., Liebesny P., Saar S. H., 2011, ApJ, 733, 115

Messina S., 2001, A\& A, 371, 1024

Morales J. C., Gallardo J., Ribas I., Jordi C., Baraffe I., Chabrier G., 2010, ApJ, 718, 502

Moraux E., Bouvier J., Clarke C., 2005, Astron. Nachr., 326, 985

Morin J., Donati J., Petit P., Delfosse X., Forveille T., Albert L., Aurière M., Cabanac R., Dintrans B., Fares R., Gastine T., Jardine M. M., Lignières F., Paletou F., Ramirez Velez J. C., Théado S., 2008, MNRAS, 390, 567

Neff J. E., O’Neal D., Saar S. H., 1995, ApJ, 452, 879

Noyes R. W., Weiss N. O., Vaughan A. H., 1984, ApJ, 287, 769

O’Dell M. A., Panagi P., Hendry M. A., Collier Cameron A., 1995, A\&A, 294, 715

O’Neal D., Neff J. E., Saar S. H., Cuntz M., 2004, AJ, 128, 1802

Pickles A. J., 1998, VizieR Online Data Catalog, 611, 863

Pizzolato N., Maggio A., Micela G., Sciortino S., Ventura P., 2003, A\&A, 397, 147

Prosser C. F., Schild R. E., Stauffer J. R., Jones B. F., 1993, PASP, 105, 269

Queloz D., Allain S., Mermilliod J.-C., Bouvier J., Mayor M., 1998, A\& A, 335, 183

Reid, M. R. 2002, Technical report, Data base of field stars. http://www.stsci.edu/ inr/cmd.html

Reiners A., Basri G., 2009, A\&A, 496, 787

Reiners A., Basri G., Browning M., 2009, ApJ, 692, 538

Ribas I., Morales J. C., Jordi C., Baraffe I., Chabrier G., Gallardo J., 2008, Memorie della Societa Astronomica Italiana, 79, 562

Rice J. B., 1996, in K. G. Strassmeier \& J. L. Linsky ed., Stellar Surface Structure Vol. 176 of IAU Symposium, Doppler imaging of stellar surfaces (review). pp 19-+

Rieke G. H., Lebofsky M. J., 1985, ApJ, 288, 618

Semel M., 1989, A\&A, 225, 456

Strassmeier K. G., 2002, Astronomische Nachrichten, 323, 309

Strassmeier K. G., 2009, A\&ARv, 17, 251

Strassmeier K. G., Rice J. B., 2006, A\&A, 460, 751

Sung H., Bessell M. S., Lee B.-W., Lee S.-G., 2002, AJ, 123, 290

Terndrup D. M., Pinsonneault M., Jeffries R. D., Ford A., Stauffer J. R., Sills A., 2002, ApJ, 576, 950

Terndrup D. M., Stauffer J. R., Pinsonneault M. H., Sills A., Yuan Y., Jones B. F., Fischer D., Krishnamurthi A., 2000, AJ, 119, 1303

Thomas J. H., Weiss N. O., 2008, Sunspots and Starspots. Cambridge University Press

van Leeuwen F., Alphenaar P., Meys J. J. M., 1987, A\& AS, 67, 483

Vogt S. S., 1981, ApJ, 250, 327

Watson C. A., Dhillon V. S., 2004, MNRAS, 351, 110

Young A., Skumanich A., MacGregor K. B., Temple S., 1990, ApJ, 349, 608 
This paper has been typeset from a $\mathrm{T}_{\mathrm{E}} \mathrm{X} / \mathrm{LT}_{\mathrm{E}} \mathrm{X}$ file prepared by the author. 